\title{
Energy-Aware Broadcasting Method for Wireless Sensor Network
}

\author{
Cheol-Min Park ${ }^{1}$, Dae-Won Kim ${ }^{1}$, and Jun Hwang ${ }^{2}$ \\ ${ }^{1}$ Chung-Ang Univ., Bobst-Hall 5 floor, System Software Lab, \\ Dept. of Computer Science \& Engineering, Seoul 221, 156-756, Republic of Korea \\ raphael66@korea.com, hide@sslab.cse.cau.ac.kr \\ ${ }^{2}$ Seoul Woman's Univ., Dept. of Information \& Media, \\ Seoul 126, 139-774, Republic of Korea \\ hjuneswu.ac.kr
}

\begin{abstract}
The communicative behaviors in Wireless Sensor Networks(WSNs) can be characterized by two different types: routing and broadcasting. The broadcasting is used for effective route discoveries and packet delivery. A blind flooding approach for broadcasting generates many redundant transmissions. The Dominant Pruning(DP) algorithm is reduced the redundant transmissions of packets based on 2-hop neighborhood information. However, in DP(include TDP/PDP) algorithm, a particular node is frequently selected as a rebroadcasting node and its life-time is shortened. As a result, DP algorithm is insufficient in terms of the overall energy dissipation in sensor network. In this paper, we propose the algorithm based on Partial Dominant Pruning(PDP) algorithm to enhance sensor network lifetime. We compare and analyze the simulation result of our algorithm with PDP.
\end{abstract}

Keywords: wireless sensor network, broadcasting, network lifetime, energyaware.

\section{Introduction}

A sensor network is composed of a large number of sensor nodes that are densely deployed either inside the phenomenon or very close to it. Each sensor node is limited in power, computational capacities, and memory size [1]. Also, as deployed sensor nodes can't replace the battery, energy efficiency is a most important factor in sensor network.

The communicative behaviors in Wireless Sensor Networks(WSNs) can be characterized by two different types: routing(node-to-sink) and broadcasting(sink-to-node or node-to-node). Broadcasting is an essential communication requirement for sink and sensor nodes. A sink node usually floods the query request to a region of all sensor nodes in a user-demand manner, asking these nodes for returning environment information. Such an application in WSNs requires a broadcasting protocol to deliver the query information from sink to all sensor nodes.

The traditional solution to the broadcasting problem is blind flooding, where each node receiving the message for the first time retransmit it to all its neighbors. Blind flooding generates many redundant transmissions. Many broadcast algorithms besides 
blind flooding have been proposed [2],[3],[4],[6],[7],[8],[9], and these algorithms utilize neighborhood and/or history information to reduce redundant packets. The dominating pruning(DP) algorithm [3] is one of the promising approaches that utilize 2-hop neighborhood information to reduce redundant transmissions. Enhancements to dominant pruning have been reported by Lou and $\mathrm{Wu}$ [2], who describe the total dominant pruning(TDP) algorithm and the partial dominant pruning(PDP) algorithm.

DP algorithm selects forwarding node set as the optimized 1-hop nodes to cover 2hop neighbor nodes to reduce the number of forwarding nodes. As a result, a particular node's lifetime is shortened because it is frequently selected as a rebroadcasting node, and it affects the overall network lifetime. Although DP algorithm selects forwarding node set as the optimized 1-hop node to cover 2-hop neighbor node, it cannot make the optimized routing path from a viewpoint of sensor network.

In this paper, we propose the algorithm to improve overall network lifetime as disperse the dissipation of node energy on sensor network using the node's energy information. Our proposed algorithm is improved the routing path for a viewpoint of sensor network by adding a flag bit to broadcasting messages. Our algorithm is based on the PDP algorithm and is modified the selection process of DP algorithm. Simulation results of applying this algorithm show that the proposed method in this paper has achieved better performance than the PDP algorithm in the lifetime of the network.

The rest of the paper organized as follow. Section 2 illustrates the TDP/PDP algorithm. The energy-aware broadcasting algorithm is proposed in Section 3 and simulation results are shown in Section 4. Conclusions are finally made in Section 5.

\section{Preliminaries}

\subsection{TDP and PDP Algorithm [2]}

We use a simple graph, $G=(V, E)$, to represent the wireless sensor network, where $V$ represent a set of wireless mobile hosts(nodes) and $E$ represents a set of edges(links). The network is seen as a unit disk graph [10], i.e., the nodes within the circle around node $v$ (corresponding to its radio range) are considered its neighbors.

We use $N(u)$ to represent the neighbor set of $u$ (including $u$ ). $N(N(u)$ represents the neighbor set of $N(u)$ (i.e., the set of nodes that are within 2-hops from $u$ ). Clearly, $\{u\} \subseteq N(u) \subseteq N(N(u))$ and if $u \in N(v)$, then $N(u) \subseteq N(N(v))$. Throughout the paper, we assume that $u$ (sender) and $v$ (receiver) are neighbors.

In DP algorithm, node $v$ just needs to determine its forwarding node list $F(u, v)$ from $B(u, v)=N(v)-N(u)$ to cover nodes in $U(u, v)=N(N(v))-N(u)-N(v)$.

In TDP Algorithm, if node $v$ can receive a packet piggybacked with $N(N(u))$ from node $\mathrm{u}$, the 2-hop neighbor set that needs to be covered by $v$ 's forward node list $F(u$, $v$ ) is reduced to $U(u, v)=N(N(v))-N(N(u))$. In the PDP algorithm, no neighborhood information of the sender is piggybacked with the broadcast packet. Therefore, the deduction of $N(N(u))$ from $N(N(v))$ cannot be done at node v. However, unlike the DP algorithm, more nodes can be excluded from $N(N(v))$. These nodes are the neighbors of each node in $N(u) \cap N(v)$. Such a node set is donated as $P(u, v)=$ $N(N(v) \cap N(u))$. Therefore, the 2-hop neighbor set $\mathrm{U}$ in the PDP algorithm is $U(u$, $v)=N(N(v))-N(u)-N(v)-P$. 
Both the TDP and PDP algorithm reduce the size of $U(u, v)$ and, hence, reduce the size of $F(u, v)$ than the original DP algorithm. But, the PDP algorithm is more cost effective, since no neighborhood information of the sender is piggybacked in the PDP during the transmission.

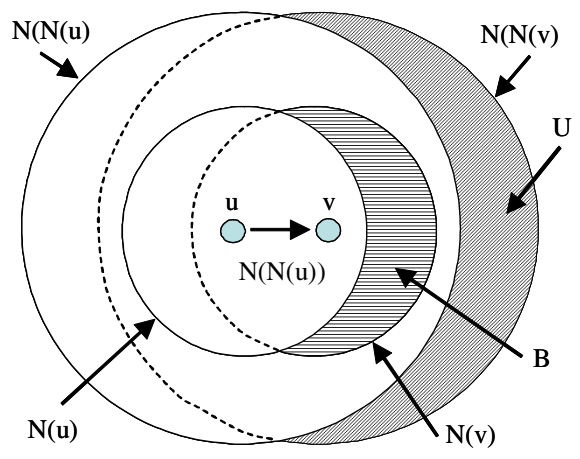

(a)

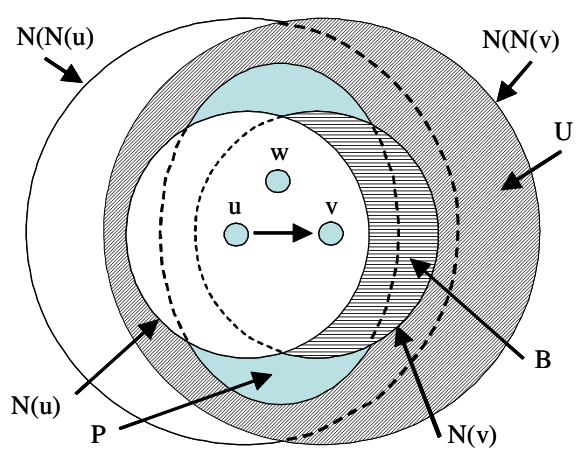

(b)

Fig. 1. Illustration for two algorithms: (a) total dominant pruning (TDP), (b) partial dominant pruning (PDP)

\subsection{Lifetime of a Sensor Network}

The definition of the lifetime of a sensor network is determined by the kind of service it provides. Hence, the lifetime of a sensor network can group into three classes.

In some cases it is necessary that all nodes stay alive as long as possible, since network quality decreases considerably as soon as one node dies. Scenarios for this case include intrusion or fire detection, and it is important to know when the first node dies.

In other cases, sensors can be placed in proximity to each other, and therefore adjacent sensors could record related or identical data. Hence, the loss of a single or few nodes does not automatically diminish the quality of service of the network. In this scenario it is needed to know the half-life period of the sensor network. Finally, for the overall lifetime of the sensor network, it is to know when the last node dies.

\section{Energy-Aware Broadcasting Protocol}

\subsection{Basic Concepts}

The PDP algorithm creates the forward node list as the optimized 1-hop nodes to cover 2-hop neighbor nodes to reduce the number of forward nodes. As a result, it is shortened a particular node's lifetime because it is frequently selected as a forward node, and it is affected the overall network lifetime. Moreover, although PDP algorithm selects the forward nodes as the optimized 1-hop neighbor nodes to cover 2-hop neighbor nodes, it can't make the optimized routing path in terms of overall sensor network.

The purpose of our proposed algorithm is to increase the sensor network lifetime. For the purpose, the algorithm selects the forward nodes taking account of node's 
energy to disperse the energy dissipation on the sensor network and separates the selection process of forwarding node list into two phases to acquire the routing path to approximate the optimal routing path.

The nodes in sensor network exchanges information and maintains neighbor node table that send/receive 'Hello' and broadcast messages. Therefore, for proposed algorithm, we add node's energy information to neighbor node table, and add sender's energy information and uncovered 2-hop node set to broadcast message.

The basic concept of algorithm is as follows. First, a node to be send message selects the forward nodes using greedy set cover algorithm[5] among nodes with energy more than the average energy of 1-hop neighbor nodes. And it broadcasts the message. Since our algorithm selects the forward nodes only to nodes more than the average energy, it occurs the uncovered 2-hop neighbor nodes when the time elapsed. In this case, the broadcast message ( $U C_{\text {node }}$ field) includes the uncovered node list. Since a node in sensor network have several neighbor nodes, the uncovered nodes in current step can be covered by another neighbor nodes at the next step. When node receives the message, if node is the forward node, it executes the forward node selection process to select next forward nodes, and broadcasts the message; otherwise, it changes the sender's energy value in neighbor node table.

Since nodes in the sensor network have several neighbor nodes, it increases the redundant transmissions if forwarding step is increased. Using CheckBit of broadcast message, our algorithm makes the routing path in terms of overall sensor network. Therefore, it can reduce the redundant transmissions.

The detailed algorithm and execution process describes next subsection.

\subsection{Algorithm Description}

Figure 2 represents a pseudo code for the proposed algorithm to create the forwarding node list. The input of the proposed algorithm is $U(u, v)$ and $B(u, v)$ to be computed by PDP algorithm, CheckBit, and the uncovered 2-hop node set $U C_{\text {node }}$ of the node $u$. The output is the forwarding node list $F$ to select by node v, modified CheckBit, and the uncovered 2-hop node set $U C_{\text {node }}$ of the node $v$. And $Z$ denotes the a subset of $U(u, v)$ covered so far, $K$ denotes the set of $S_{i}$, and $S_{i}$ denotes the neighbor set of $v_{i}$ in $U(u, v)$. In this place, $u$ is sender and $v$ is receiver. Table 1 summarizes these terminologies.

The selection process of forwarding node list separates into two phases using CheckBit. In the first phase $($ CheckBit $=0)$, it selects the forward nodes among nodes more than the average energy of 1-hop neighbor nodes. Set CheckBit $=1$, and add the forward node set to packet. In the other phase $($ CheckBit $=1)$, it selects the forward node to one node that has the maximum number of uncovered neighbors in 2-hop neighbor nodes among nodes more than the average energy of 1-hop neighbor nodes. Set CheckBit $=0$, and add a forward node to packet.

The step by step description is provided as follows.

Step 1 (Line 01-02) Initialize $F, Z$, and $K$ to use in algorithm. Add the node set to be covered by received node and the node set that the sender is not covered

Step 2 (Line 03-06) For $v_{i} \in B(u, v)$, if energy of node $v_{i}$ is more than the average energy of $N(v)$, find the intersection $S_{i}$ of $N\left(v_{i}\right)$ and $U(u, v)$, and add to $K$. 


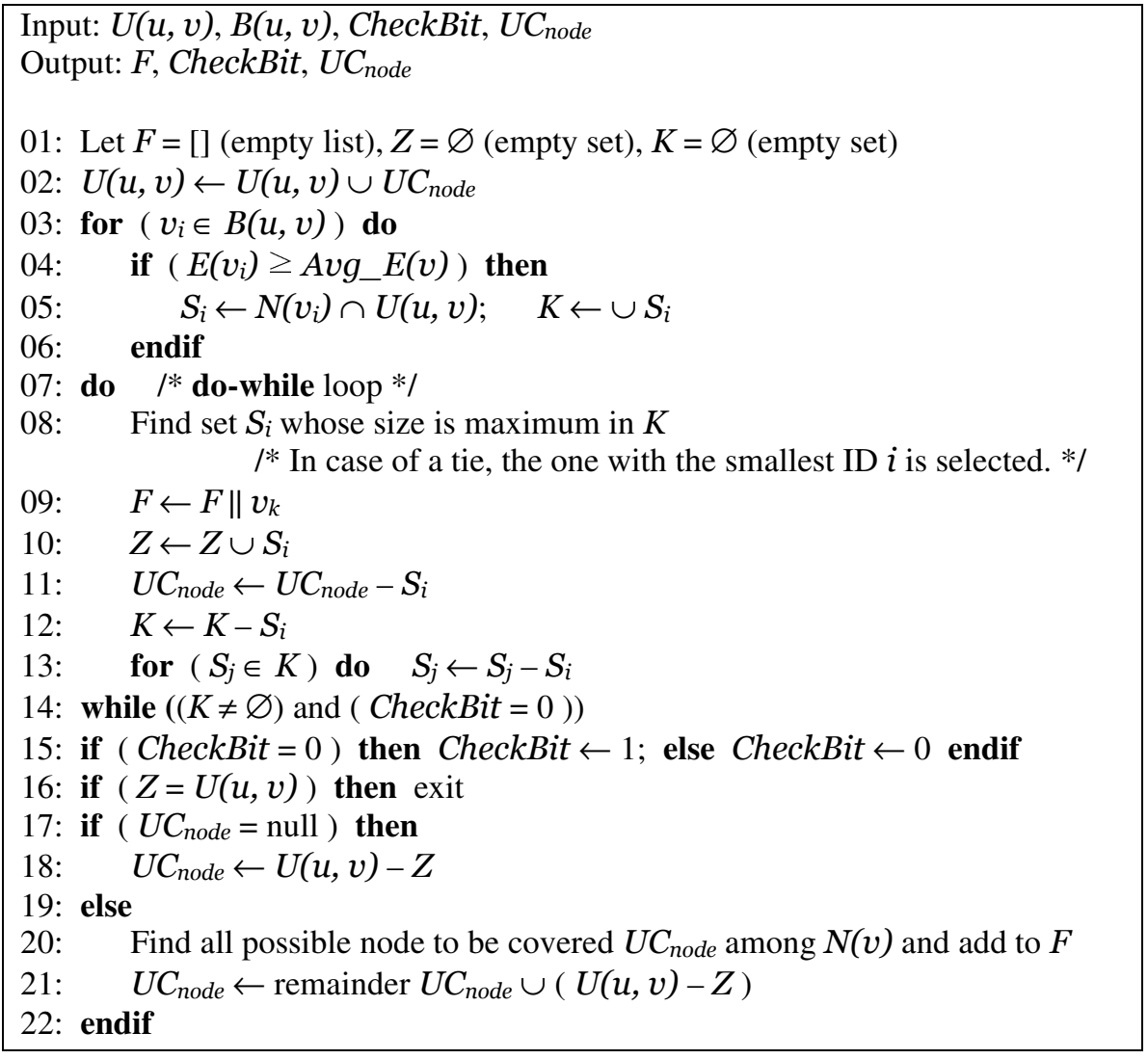

Fig. 2. Pseudo code of the proposed algorithm

Table 1. Notation

\begin{tabular}{|ll|}
\hline Notation & Meaning \\
\hline$U(u, v)$ & the node set to be covered by node $v$ \\
$B(u, v)$ & Potential forward node set to cover $U(u, v)$ \\
$U C_{\text {node }}$ & the node set in $N(N(u))$ to uncovered node by node $u$ \\
$F$ & forward node list \\
$Z$ & a subset of $U(u, v)$ covered so far \\
$S_{i}$ & the neighbor set of $v_{i}$ in $U(u, v)$ \\
$K$ & the set of $S_{i}$ \\
$A v g_{-} E(v)$ & Average energy of $N(v)$ \\
$E\left(v_{i}\right)$ & energy of node $v_{i}$ \\
\hline
\end{tabular}

Step 3 (Line 08-13) Find set $S_{i}$ whose size is maximum in $K$ (in case a tie, the one with smallest ID $i$ is selected). Add node with the detected $S_{i}$ to the forward node list $F$, and adds the detected $S_{i}$ to a covered node set $Z$. Removes the 
detected $S_{i}$ from the uncovered 2-hop node set $U C_{\text {node }}$ of the sender and $K$. Remove $S_{i}$ from the remainder subsets of $K$.

Step 4 (Line 14) If $K$ is empty or CheckBit is 1, it breaks do-while loop (i.e., dowhile loop (line 07-14) executes once only and one node selects the forward node). Otherwise, repeat step 3 until $K$ is empty.

Step 5 (Line 15) Change the received CheckBit value.

Step 6 (Line 16) If all 2-hop neighbor nodes is covered, the algorithm is finished.

Step 7 (Line 17-18) If $U C_{\text {node }}$ of the sender is null, add uncovered 2-hop neighbor node to $U C_{\text {node. }}$.

Step 8 (Line 19-22) Find 1-hop neighbor nodes whose can cover $U C_{n o d e}$ regardless of node's energy, add it to $F$, and remove covered node from $U C_{\text {node }}$. Add uncovered 2-hop neighbor nodes to $U C_{\text {node }}$.

\section{Simulations}

In this section, we evaluate the performance of the PDP algorithm and our algorithm in terms of the following evaluation bases: reachability (the number of all sensors receiving a packet), the number of forward nodes, the average number of packets a node receives, and the network lifetime (the number of alive sensor nodes).

The simulator randomly generates a connected unit disk graph within a broadcast area of $m \times m$ (with $m=100$ ). Graphs are generated in two ways: a fixed transmitter range $(r)$ and a fixed average node degree $(d)$. The average node degree is the expected number of nodes that are within a node's transmitter range. Specially, the average node degree can be approximated as $d=\left(\pi r^{2} / m^{2}\right) \times n$ [2]. The number of hosts is 30,60, 90 and 120. The node transmitter range is supposed 30, 40, 50 and 60 . The simulation is conducted under the static environment. Assumed that dissipate the node's energy only when send or receive the messages (packets).

When a source node broadcasts a packet(broadcast message), each intermediate node will decide whether to retransmit the packet or to drop it independently, based on a given termination criterion. In other words, the broadcast process at each node will terminate when a given termination criterion is satisfied. In this paper, we suppose the following termination criteria. (Since each termination is decided locally, this approach corresponds to a reasonable termination criterion in a real system.)

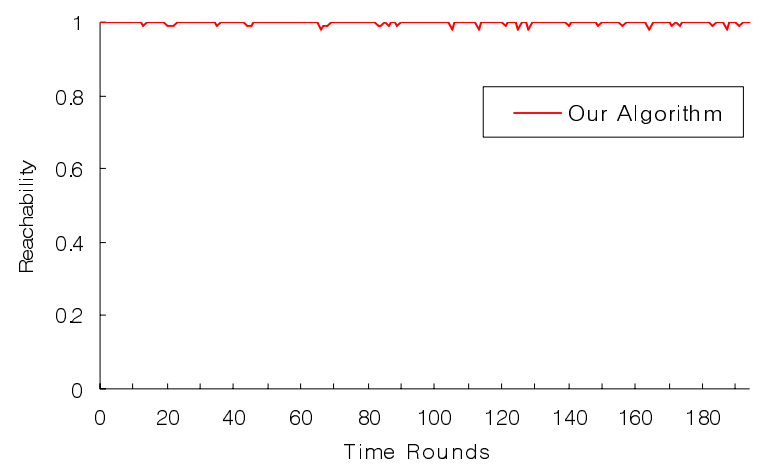

Fig. 3. The reachability of proposed algorithm 

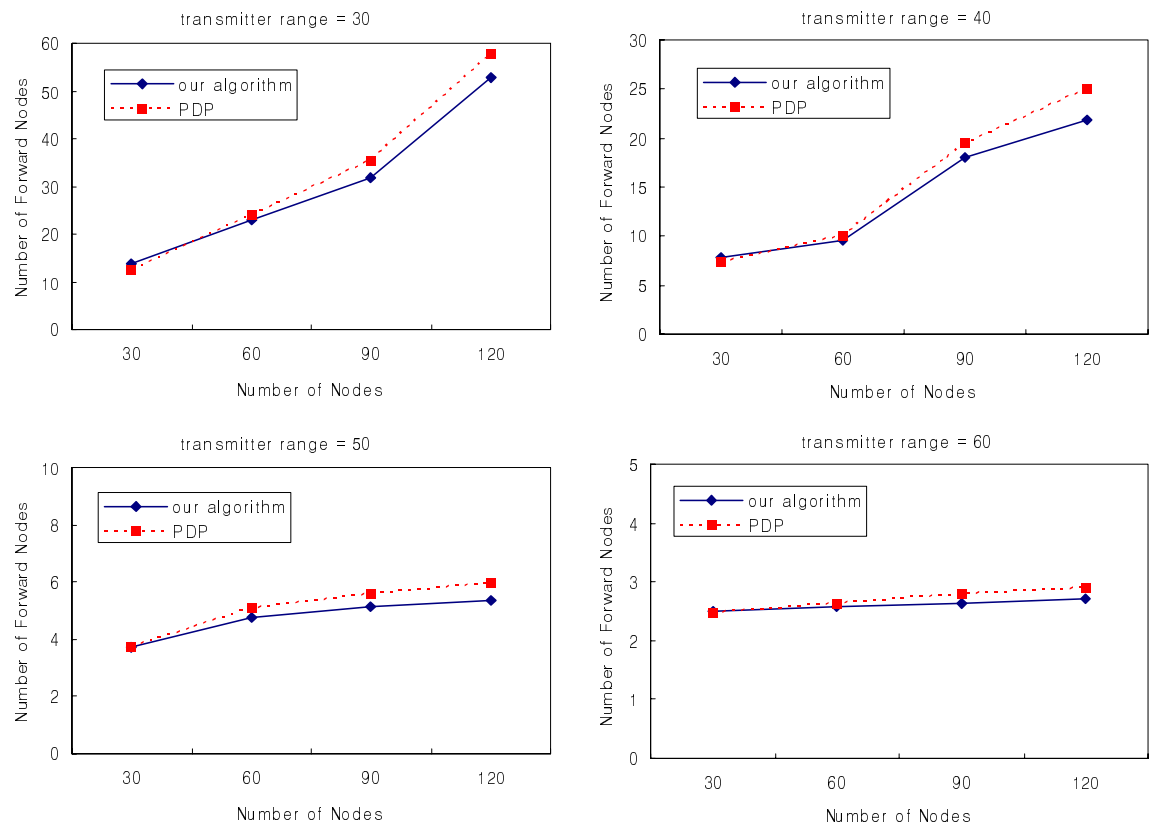

Fig. 4. The average number of forward nodes with the relayed/un-relayed criterion
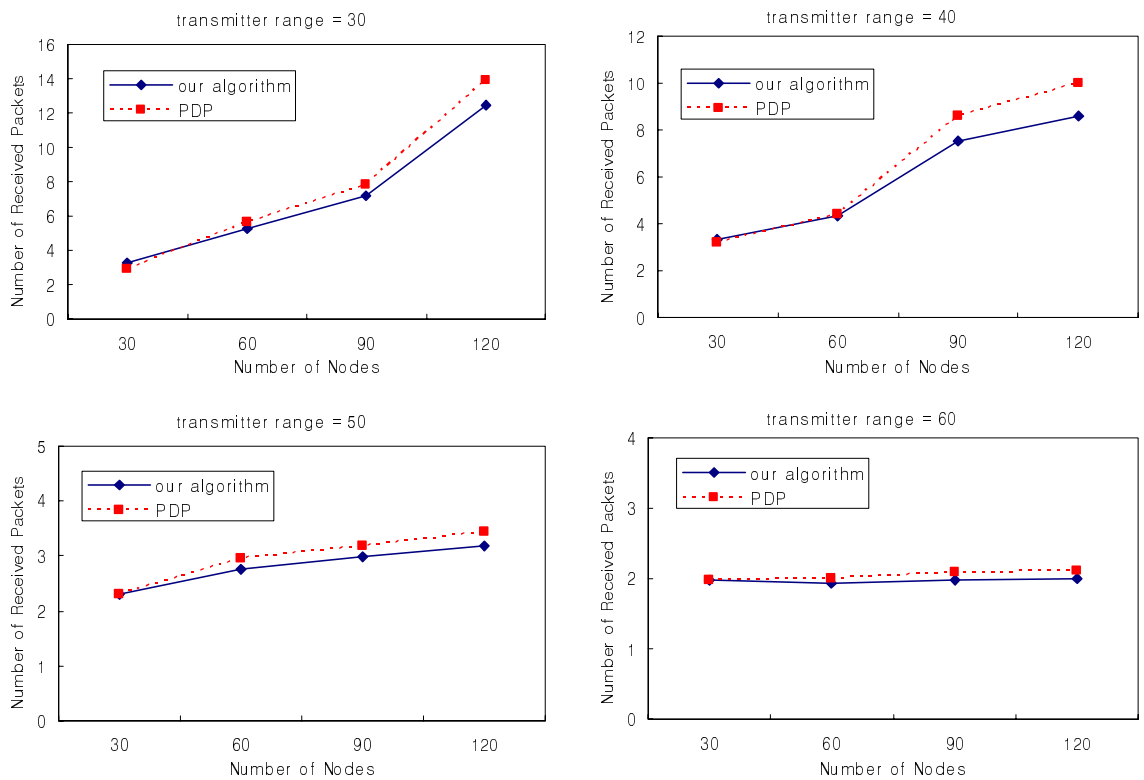

Fig. 5. The average number of packets a node receives with the relayed/un-relayed criterion 
- The broadcast process (including the forward node selection and the broadcast process itself) is done quickly so that $N(v)$ and $N(N(v))$ remain the same during the process for each host $v$.

- Each node assigns a relayed/un-relayed status. A node $v$ is called relayed when $v$ has a packet; otherwise, $v$ is called un-relayed.

- Forward node $v$ will stop rebroadcasting a packet if $v$ is relayed status.

Figure 3 shows the reachability of our algorithm. Figure 4 shows the average numbers of forward nodes and figure 5 shows the average numbers of packets a node receives during the broadcast process under different algorithms. Also, Figure 6 shows the network lifetime under different algorithms. (Figure 4 and 5 is the simulation results under all nodes alive.)
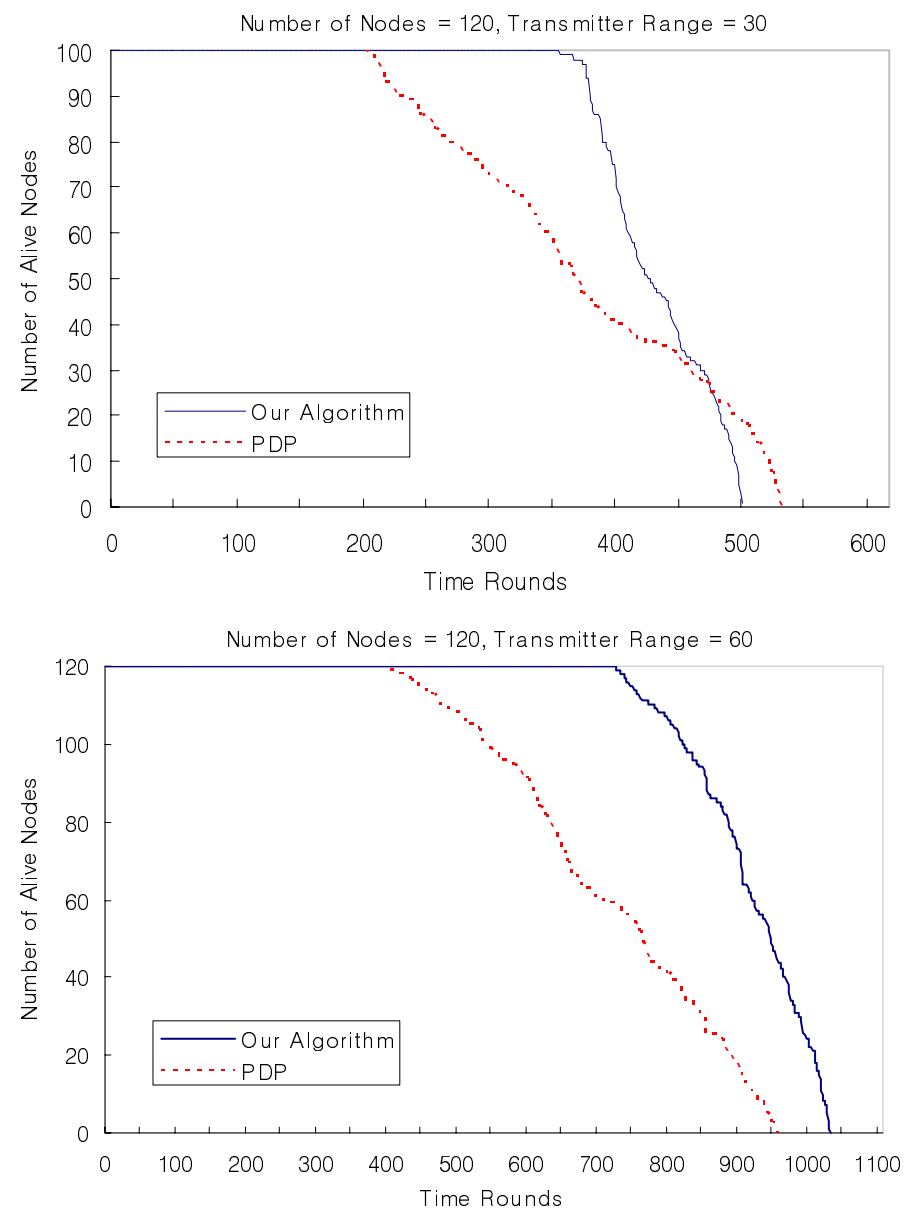

Fig. 6. Network lifetime (the number of alive nodes) 
Figure 3 is the simulation results on reliability among performance evaluation criterion of broadcast protocol. The reliability represents reachability, i.e., the ratio of nodes connected to the source that received the broadcast message. The proposed algorithm records about $100 \%$ in terms of the message reception rate.

Figure 4 and 5 show the simulation results of the average number of forward nodes and the average number of broadcast packets that a node receives during the broadcast process for the fixed number of node $(30,60,90$ and 120), under relayed/un-relayed termination criteria. Our proposed algorithm shows the performance better than PDP algorithm when the number of nodes and transmitter range is increases. But, when the node density is low, our algorithm shows the performance same or less than PDP algorithm.

Figure 6 is the simulation results of network lifetime based on network transmitter range. As mentioned early (section 2.2), the lifetime of the sensor network can group into three classes by the kind of service it provides; when the first node dies, the halflife period of a sensor network, and the overall lifetime of a sensor network. In figure 6 , it knows that the performance of our algorithm is better than PDP algorithm.

\section{Conclusion}

In this paper, we introduced a broadcast method that prolongs the network lifetime. For increase the sensor network lifetime, our proposed algorithm selects the forward nodes taking account of node's energy to disperse the energy dissipation on the sensor network and separates the selection process of forwarding node list into two phases to acquire the routing path to approximate the optimal routing path. Simulation results of applying this algorithm show that the proposed method in this paper has achieved better performance than the PDP algorithm in the lifetime of the network.

Acknowledgments. This paper was supported by special research funds of Seoul Woman's University in 2005.

\section{References}

1. Ian F. Akyildiz, Weilian Su, Yogesh Sankarasubramaniam, and Erdal Cayirci, "A Survey on Sensor Networks", IEEE Communications Magazine, pp.102-114, Aug. 2002.

2. Wei Lou and Jie Wu, "On Reducing Broadcast Redundancy in Ad Hoc Wireless Networks", Proc. HICSS'03, pp.305b, Jan. 2003.

3. H. Lim and C. Kim, "Flooding in wireless ad hoc networks", Computer Communications Journal, 24(3-4):353-363, 2001.

4. Ivan Stojmenovic and Jie Wu, "Broadcasting and Activity-Scheduling in Ad Hoc Networks", Mobile Ah Hoc Networking, IEEE/Wiley, pp.205-229, 2004.

5. L. Lovasz, "On the ratio of optimal integral and fractional covers", Discrete Mathematics, vol. 13, pp. 383-390, 1975.

6. K. M. Alzoubi, P. J. Wan, and O. Frieder, "New distributed algorithm for connected dominating set in wireless ad hoc networks", Proc. HICSS-35, Jan. 2002. 
7. G. Calinescu, I. Mandoiu, P. J. Wan, and A. Zelikovsky, "Selecting forwarding neighbors in wireless ad hoc networks", Proc. ACM DIALM'2001, pp.34-43, Dec. 2001.

8. A. Qayyum, L. Viennot, and A. Laouiti, "Multipoint relaying for floording broadcast message in mobile wireless networks", Proc. HICSS-35, Jan. 2002.

9. I. Stojmenovic, S. Seddigh, and J. Zunic, "Dominating sets and neighbor elimination based broadcasting algorithms in wireless networks", IEEE Trans. on Parallel and Distributed Systems, 13(1):14-25, Jan. 2002.

10. B. N. Clark, C. J. Colbourn, and D. S. Johnson, "Unit Disk Graph”, Discrete Mathematics, vol. 86, pp. 165-177, 1990. 